\title{
Classification of breast lesions based on a dual S-shaped logistic model in dynamic contrast enhanced magnetic resonance imaging
}

\author{
DANG Yi ${ }^{1}$, GUO Li ${ }^{2}$, LV DongJiao ${ }^{3}$, WANG XiaoYing ${ }^{1,2^{*}} \&$ ZHANG Jue ${ }^{1,3^{*}}$ \\ ${ }^{1}$ Academy for Advanced Interdisciplinary Studies, Peking University, Beijing 100871, China; \\ ${ }^{2}$ Department of Radiology, Peking University First Hospital, Beijing 100034, China; \\ ${ }^{3}$ College of Engineering, Peking University, Beijing 100871, China \\ Received July 13, 2011; accepted August 9, 2011
}

\begin{abstract}
This study proposes a novel dual S-shaped logistic model for automatically quantifying the characteristic kinetic curves of breast lesions and for distinguishing malignant from benign breast tumors on dynamic contrast enhanced (DCE) magnetic resonance (MR) images. $\mathrm{D}(\alpha, \beta)$ is the diagnostic parameter derived from the logistic model. Significant differences were found in $\mathrm{D}(\alpha, \beta)$ between the malignant benign groups. Fisher's Linear Discriminant analysis correctly classified more than $90 \%$ of the benign and malignant kinetic breast data using the derived diagnostic parameter $(\mathrm{D}(\alpha, \beta))$. Receiver operating characteristic curve analysis of the derived diagnostic parameter $(\mathrm{D}(\alpha, \beta))$ indicated high sensitivity and specificity to differentiate malignancy from benignancy. The dual S-shaped logistic model was effectively used to fit the kinetic curves of breast lesions in DCE-MR. Separation between benign and malignant breast lesions was achieved with sufficient accuracy by using the derived diagnostic parameter $\mathrm{D}(\alpha, \beta)$ as the lesion's feature. The proposed method therefore has the potential for computer-aided diagnosis in breast tumors.
\end{abstract}

logistic model, breast cancer, dynamic contrast enhanced magnetic resonance imaging

Citation: Dang Y, Guo L, Lv D J, et al. Classification of breast lesions based on a dual S-shaped logistic model in dynamic contrast enhanced magnetic resonance imaging . Sci China Life Sci, 2011, 54: 889-896, doi: 10.1007/s11427-011-4221-7

Breast cancer is one of the most frequently diagnosed cancers in women, with a mortality rate second only to lung cancer [1]. Each year, over 1.2 million women develop invasive breast cancer and more than 500000 die of this disease worldwide. With the rapid advancement of medical technology, especially in functional imaging and evaluation methods, the mortality rate of breast cancer has experienced a steady decrease of $3.2 \%$ per year among women less than 50 years of age, and $2.0 \%$ among women greater than 50 years of age, since 1990 [2]. Still, effective early detection of breast cancer that aims to identify lesions before symptoms develop is a key factor in the continual improvement

*Corresponding author (email: cjr.wangxiaoying@vip.163.com; zhangjue@pku.edu.cn) of survival rates.

Dynamic contrast enhanced magnetic resonance imaging (DCE-MRI) is a functional evaluation approach that characterizes blood vessels and tissues by comparing magnetic resonance images acquired before and after intravenous injection of the contrast agent gadolinium. Through a series of key studies, it has gradually been recognized as serving an important role in quantitative functional evaluation of breast cancer. For example, DCE-MRI has been used to investigate suspicious micro calcifications either in isolation or associated with a breast mass on mammography [3,4], to stage loco-regional tumors in patients with primary malignancy [5], and to predict and monitor pretreatment tumor response to neo-adjuvant chemotherapy [6-8]. In addition, 
DCE-MRI has the potential to differentiate between malignant and benign lesions [9-12]. Because of the complexity involved in interpreting the data, effective quantification of DCE-MRI is crucial to accurate diagnosis, and treatment evaluation of breast cancer in the clinical setting.

Most of the existing DCE-MRI quantitative evaluation methods examine the kinetic curves formed by connecting the average enhancement over time within a region of interest (ROI) defined by a human observer over the suspected lesion area. Previous DCE-MRI data analysis approaches include pharmacokinetics model and kinetic enhancement curve fitting.

Pharmacokinetics models, constructed upon the filtration of contrast agents into lesion tissues, retrieve physiological information such as blood perfusion, capillary leakage, water exchange rate, diffusion and microvasculature from the characteristics of the kinetic curves [13-15]. In the model proposed by Tofts and Kermode [13], physiological parameters are extracted such as the transcapillary transfer from plasma into interstitial space $\left(K^{\text {trans }}\right)$, the efflux transcapillary transfer rate constant $\left(K_{\mathrm{ep}}\right)$, and the volume of extravascular extracellular space (EES) per unit volume of tissue $\left(V_{\mathrm{e}}\right)$. In this approach, pathological patterns of the lesions are reflected in the physiological features of the contrast agent kinetics, independent of imaging conditions. However, pharmacokinetics models based on feature extraction methods have their own limitations. For instance, they do not effectively address pressure gradients associated with fluid and solute transport in tissues, which may bring significant deviations when the model is applied to tumors exhibiting high interstitial fluid pressure (IFP) [16]. Also, the difficulty in drawing the baseline $\mathrm{T} 1$ relaxation rate, which is a key factor in converting signal intensity to contrast agent concentration, introduces errors. In addition, the estimation of the arterial input function (AIF) involved in signal intensity conversion varies for different ROI locations, causing discrepancies among different physiological models.

Kinetic curve fitting approaches are based upon the relationship between the shape of the kinetic curves and the degree of malignancy of the lesions. The breast imaging reporting and data system (BI-RADS) lexicon [17] categorized kinetic enhancement curves into three types based on their initial rise and delayed phase (type I: persistent increase; type II: maintains a plateau after initial increase; type III: rapid washout after initial increase). A previous study [18] showed that most benign lesions always maintain successive enhancement during the measured time course (type I) whereas malignant lesions frequently demonstrate rapid washout after the initial increase (type III). In the semi-quantitative model proposed by Szabo et al. [19], the maximum contrast enhancement, time to peak enhancement, and the descriptor of margins were extracted to distinguish between malignant and benign lesions. Unfortunately, this method is susceptible to noise [20], and inconsistencies be- tween the parameters acquired under different experimental conditions have affected its clinical viability. Heiburg et al. [21] applied a fifth-order polynomial to simulate DCE-MRI data. A logistic model [22] was introduced by Moate et al. for kinetic curve fitting. These proposed models well predicted all three types of kinetic curves categorized by the BI-RADS lexicon, and were verified against clinic diagnostic values of the parameters in the models. However, no quantitative results have been given to support the feasibility of this approach in differentiating between malignant and benign lesions.

In this retrospective study, a modified, 4-variable quantitative model derived from a classical logistic equation (dual S-shaped logistic model) is introduced to investigate DCE-MRI kinetic curves of breast lesions. Fitting parameters $\alpha$ and $\beta$ were extracted in an attempt to distinguish between malignant and benign lesions in 2-D parameter space. Sensitivity and specificity to malignant lesions using the derived diagnostic parameter $\mathrm{D}(\alpha, \beta)$ was evaluated by using receiver operating characteristic (ROC) analysis.

\section{Materials and methods}

\subsection{Patient population}

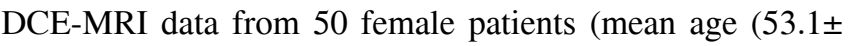
10.6) years, range 29-70 years) were acquired consecutively between August 2008 and June 2011 as approved by the local ethics committee. Analysis was performed retrospectively. Based on the consensus opinion of two pathologists and two radiologists, each with three years of experience, there were 25 benign and 25 malignant lesions investigated in this study. The diagnoses of all 50 subjects were confirmed pathologically.

\subsection{MR imaging protocol}

Subjects were scanned in a prone position using a standard double-breast coil on a $1.5 \mathrm{~T}$ whole-body MRI system (Signa; GE Medical Systems, Milwaukee, Wisconsin, USA). T1-weighted 3D spoiled gradient echo sequence with fat suppression was used in the DCEMR imaging using the following parameters: repetition time (TR), $6.9 \mathrm{~ms}$, echotime (TE), $2.7 \mathrm{~ms}$, flip angle, $15^{\circ}$; field of view (FOV), 320 $\mathrm{mm} \times 320 \mathrm{~mm}$; matrix, $512 \times 512$; slice thickness, $2.4 \mathrm{~mm}$. After the acquisition of the pre-contrast series, gadolinium-diethylene triamine pentaacetic acid (Gd-DTPA) as the contrast agent was delivered intravenously by power injection with a dose of $0.2 \mathrm{~mL} \mathrm{~km}^{-1}$ body weight and a flow rate of $2 \mathrm{~mL} \mathrm{~s}^{-1}$.

\subsection{ROI analysis}

To generate the kinetic curve, a 9-pixel square mask was 
used to select the area which was perceived to be the most enhancing part of the lesion on the first post-contrast image. The average signal intensity versus time was then calculated, yielding six data points on the kinetic curve of each ROI.

\subsection{Time phase}

Six to eight time phases of post-contrast images were acquired after pre-contrast images and one time phase obtained before injection, resulting in 7-9 data points on each kinetic curve. The scan time of each time phase was $60 \mathrm{~s}$ and there was no interval between time phases.

\subsection{Dual S-shaped logistic model}

A standard logistic model (eq. (1)) was used to describe the growth of a biotic population:

$$
S I(t)=\frac{1}{1+C e^{-k t}},
$$

where $S I$ is the signal intensity at time $t$ and $C$ is the amplitude of the plateau of the curve. This model is able to predict persistent increase (type I) and sigmoid (type II) growth patterns. In this dual S-shaped logistic model, an additional term $\left(1+m e^{-\alpha t}\right)$ is introduced to fit the washout phase of type III (eq. (2)):

$$
S I(t)=\frac{1+m e^{-\alpha t}}{1+n e^{-\beta t}},
$$

where $S I$ is the signal intensity, $\alpha\left(\mathrm{min}^{-1}\right)$ denotes the rate of signal decrease, $\beta\left(\mathrm{min}^{-1}\right)$ represents the rate of signal increase, $m$ and $n$ are related to the upper and lower limit of the signal intensity, and $t$ refers to the time phase of imaging, which ranges from 1 to 6 . Based on this proposed model, unconstrained nonlinear optimization was performed to generate coefficients $(m, n, \alpha$ and $\beta$ ) to fit the kinetic curves.

Because different patterns of kinetic curves reflect various histopathologic properties of the breast lesion [23-26], $\alpha$ and $\beta$ denoting the rate of signal decrease and signal increase, respectively, are the main factor influencing the pattern of the kinetic curve. Therefore, we extracted $\alpha$ and $\beta$ as 2 -D features of the breast lesion. The feature parameters $(\alpha$ and $\beta)$ were combined as $\mathrm{D}(\alpha, \beta)$ by using a linear equation.

The most widely described features of DCE MRI analysis are the uptake and wash-out patterns of the contrast agent in regions of suspected lesion [18]. Therefore, the up-slope (the maximum point-to-point enhancement slope) and tail-slope (the slope of a regression over the final few time-points) of kinetic curves were calculated to compare the diagnostic accuracy with the diagnostic parameter $\mathrm{D}(\alpha$, $\beta$ ) derived from our model.

\subsection{Statistical analysis}

The fitting coefficient $R^{2}$ was calculated for each patient as follows (eq. (3)):

$$
R^{2}=1-\frac{\sum_{i=1}^{N}\left(S I_{\mathrm{O} i}-S I_{\mathrm{F} i}\right)^{2}}{\sum_{i=1}^{N}\left(S I_{\mathrm{O} i}-\overline{S I}_{\mathrm{O}}\right)^{2}},
$$

where $S I_{\mathrm{O} i}$ and $S I_{\mathrm{Fi}}$ are the signal intensities of the original kinetic curve and the fitted curve, respectively. $\overline{S I}_{O}$ is the mean value of $S I_{\mathrm{O} i}$ and $N$ is the number of the time phase.

An independent samples $t$-test was performed between two groups for each variable ( $\mathrm{D}(\alpha, \beta)$, up-slope and tail-slope).

Fisher's Linear Discriminant (FLD) analysis was used to classify the kinetic curves into two groups (benign and malignant), in which the original multi-dimensional dataset was projected onto a one-dimensional straight line to maximize the inter-class distance and minimize the distance between class members. The separation $S$ between these two classes is defined as the ratio of the variance between the classes $\left(\sigma_{\text {between }}^{2}\right)$ to the variance within the classes $\left(\sigma_{\text {within }}^{2}\right)$ (eq. (4)):

$$
\begin{aligned}
S & =\frac{\sigma_{\text {between }}^{2}}{\sigma_{\text {within }}^{2}}=\frac{\left(\vec{\omega} \cdot \vec{\mu}_{y=1}-\vec{\omega} \cdot \vec{\mu}_{y=0}\right)^{2}}{\vec{\omega}^{T} \sum_{y=1} \vec{\omega}+\vec{\omega}^{T} \sum_{y=0} \vec{\omega}} \\
& =\frac{\left(\vec{\omega} \cdot\left(\vec{\mu}_{y=1}-\vec{\mu}_{y=0}\right)\right)^{2}}{\vec{\omega}\left(\sum_{y=0}+\sum_{y=1}\right) \vec{\omega}},
\end{aligned}
$$

where $\vec{\mu}_{y}=0, \vec{\mu}_{y}=1$ and $\sum_{y=0}, \sum_{y=1}$ are the means and covariances of two classes, respectively. $\vec{\omega}=\vec{\mu}_{y=i}$ is the mean and $\vec{\omega}^{T} \sum_{y=i} \vec{\omega}$ is the variance for $i=0,1$ of the linear combination of features $\vec{\omega} \cdot \vec{x}$.

Classification performance and diagnostic accuracy for the combined parameter $\mathrm{D}(\alpha, \beta)$ and empirical parameters (up-slope and tail-slope) were evaluated by Receiver Operating Characteristic (ROC) analysis. The area under the ROC curve (AUC) was used as an index to evaluate the inherent distinguishing capacity among benign and malignant lesions. A $P$-value of less than 0.05 was considered statistically significant. Sensitivity and specificity were calculated to evaluate the performance of a binary classification test.

\subsection{Steps involved in our dual S-shaped logistic model}

(i) The average DCE-MRI signal intensity as a function of time $(S I(t))$ in the selected ROI was calculated.

(ii) Convert signal to relative enhancement (ignoring $\mathrm{T} 1$ issues): signal/baseline-1.

(iii) Fit the kinetic curve SI using the dual S-shaped lo- 
gistic model following eq. (1). The initial values of $\alpha, \beta, m$ and $n$ were $-0.1,2,1,1$. Unconstrained nonlinear optimization was used to find the minimum of a scalar function of several variables, starting at the initial estimate.

(iv) Extract $\alpha$ and $\beta$ as characteristic features of the curve, which reveal the rate of signal increase and the rate of signal decrease.

(v) Map all curves onto points in 2-D parameter space by using their features ( $\alpha$ and $\beta$ ) as coordinates.

(vi) Classify the points assuming a binary outcome (benign and malignant).

(vii) Calculate the up-slope (the maximum point-to-point slope) and tail-slope (the slope of a regression over the final three time-points) of the kinetic curves.

(viii) Classification performance and diagnostic accuracy for the $\mathrm{D}(\alpha, \beta)$, up-slope and tail-slope were evaluated by ROC analysis.

\section{Results}

To obtain a prediction of a kinetic curve's type, feature parameters $(\alpha$ and $\beta$ ) for the curve were entered into a linear regression model. On the basis of Fisher's Linear Discrimi-

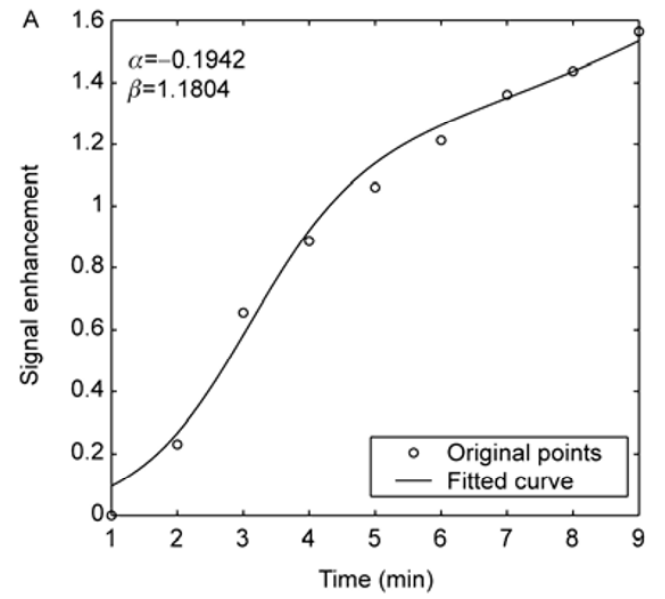

nant analysis of this model, the equation for the probable type of a kinetic curve was obtained as follows:

$$
\mathrm{D}(\alpha, \beta)=0.7079 \times \alpha+0.2921 \times \beta .
$$

In our study, 14 malignant lesions were associated with type III curves (washout) which exhibit a decreasing pattern. Eight malignant lesions and six benign lesions were associated with type II curves (plateau) which reach a plateau after an initial increase. Nineteen identified benign lesions and three malignant lesions were associated with type I (persistent) curves which demonstrate a continuous increase in enhancement with the measurement time.

The curves of all 50 subjects (22 type I, 14 type II and 14 type III), fitted with the dual S-shaped logistic model, yielded an average fitting coefficient $R^{2}$ of 0.98 for type I (Figure 1A), 0.96 for type II (Figure 1B) and 0.93 for type III (Figure 1C).

Figure 2A illustrates the statistical distribution of the combined parameter $\mathrm{D}(\alpha, \beta)$. Table 1 gives the means and standard deviations (SDs) of $\mathrm{D}(\alpha, \beta)$, up-slope and tail-slope for the ROIs, which reveal that the mean values of $\mathrm{D}(\alpha, \beta)$ and up-slope of the malignant ROIs are consistently higher than the control cases at a confidence interval of $95 \%$.
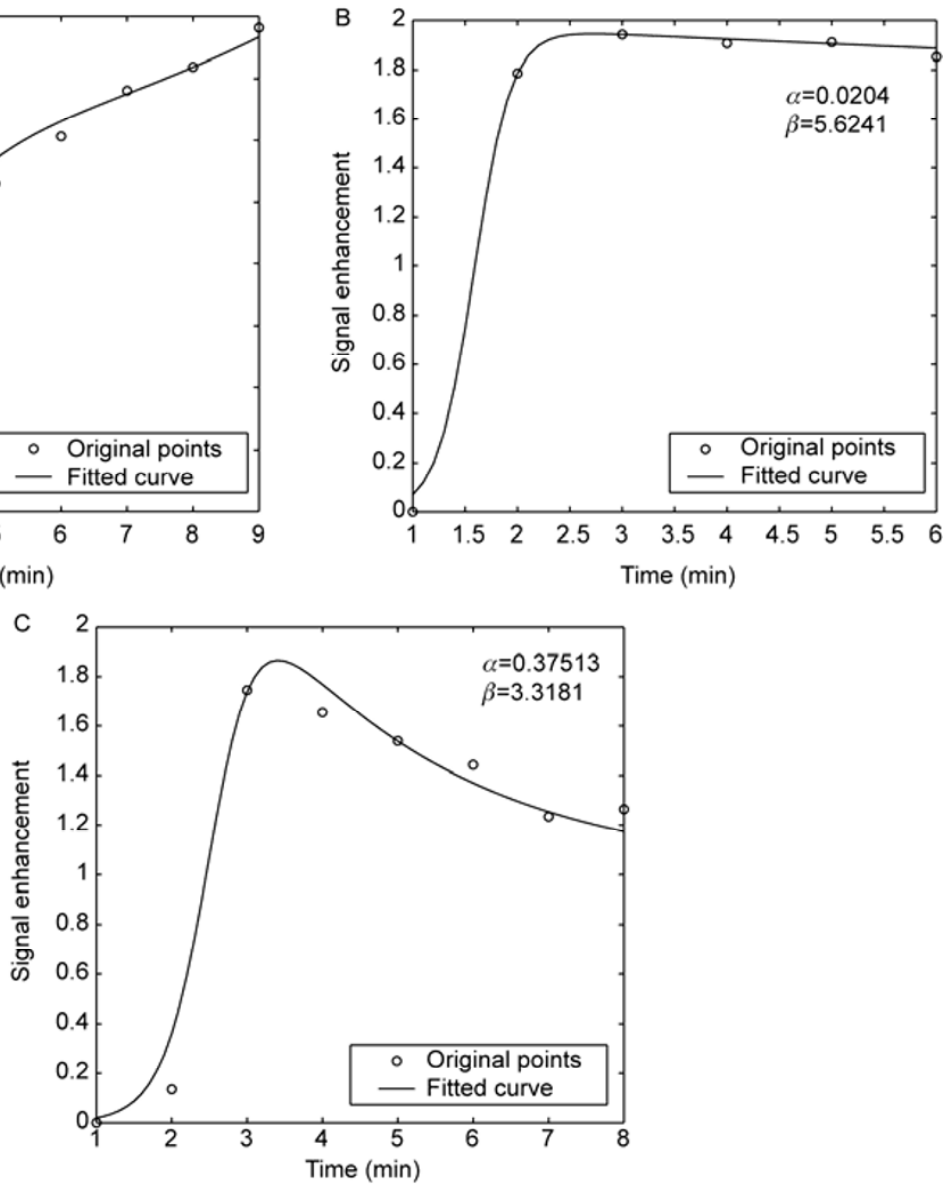

Figure 1 Typical type I kinetic curve of benign lesions (A), typical type II kinetic curve (B) and type III kinetic curve (C) of malignant lesions fitted using the dual S-shaped logistic model. 

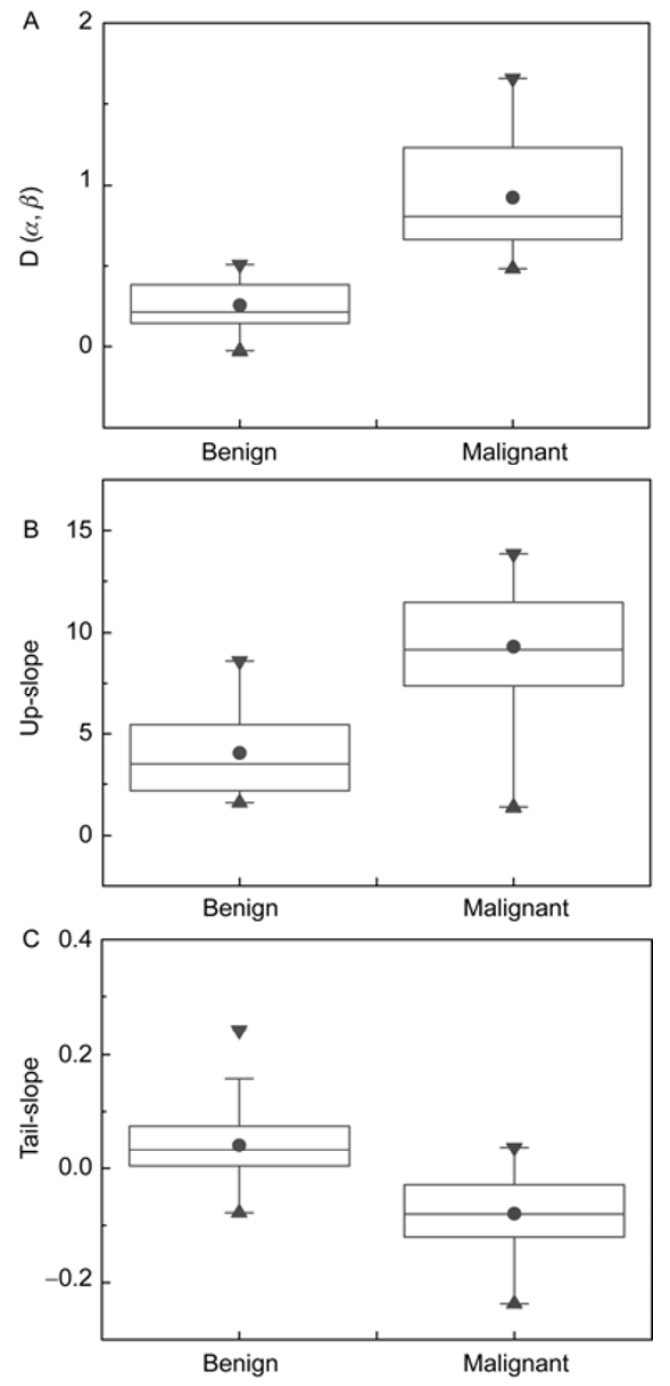

Figure 2 Box-plots of statistical distributions for $\mathrm{D}(\alpha, \beta)$ (A), up-slope (B) and tail-slope (C) in benign lesions and malignant lesions. Lines in the boxes represent median values. Whiskers extended from the boxes indicate the range of $1.5 \times$ interquartile range (IQR) of the data.

Table 1 The means and SDs of $\mathrm{D}(\alpha, \beta)$, up-slope and tail-slope in benign (25 ROIs) and malignant (25 ROIs) ROIs ${ }^{\text {a) }}$

\begin{tabular}{cccc}
\hline $\begin{array}{c}\text { Extracted } \\
\text { parameters }\end{array}$ & Benign (ROIs) & Malignant (ROIs) & $P$-value \\
\hline $\mathrm{D}(\alpha, \beta)$ & $1.046 \pm 0.425$ & $2.782 \pm 0.109$ & $<0.001$ \\
Up-slope & $4.043 \pm 2.104$ & $9.304 \pm 2.904$ & $<0.001$ \\
Tail-slope & $0.041 \pm 0.072$ & $-0.079 \pm 0.071$ & $<0.001$ \\
\hline
\end{tabular}

a) $P$-values calculated with two-sample $t$-tests were used to identify significant differences between each variable obtained in benign and malignant regions of interest (ROIs). Data presented are mean \pm standard deviation (SD).

Group differences were observed for $\mathrm{D}(\alpha, \beta)(P<0.001)$, up-slope and tail-slope (Table 1). For all 50 subjects, FLD was used as a classifier, in which more than $90 \%$ of the benign and malignant ROI data were classified correctly. The clear partition (Figure 3) indicated that the data for the benign and malignant ROIs were well separated in parameter space.
To evaluate diagnostic performance, ROC curves were generated for all parameters as shown in Figure 4, with calculated $A_{z}$ (area under the curve) values (Table 2). The parameter tail-slope had the smallest area under the ROC curve $\left(A_{2}=0.104\right)$, while the combined parameter $\mathrm{D}(\alpha, \beta)$ had the largest $\left(A_{z}=0.996\right)$. From these ROC curves we can see that the sensitivity and specificity of $\mathrm{D}(\alpha, \beta)$ to classify malignant lesions was better than that of the up-slope and

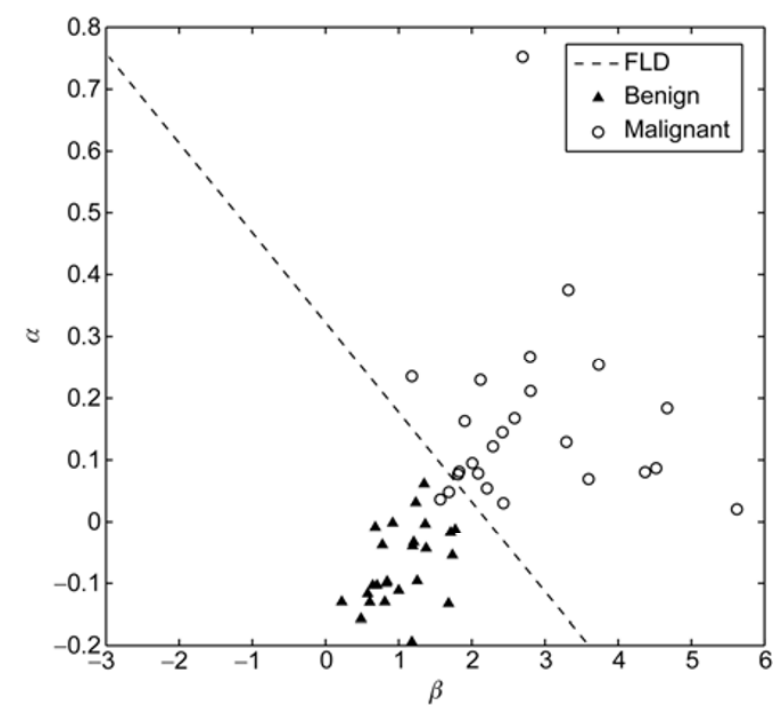

Figure 3 Classification result of benign and malignant lesions' kinetic curves using Fisher's Linear Discriminant (FLD) with $\alpha$ and $\beta$ as the abscissa and ordinate axes, respectively.

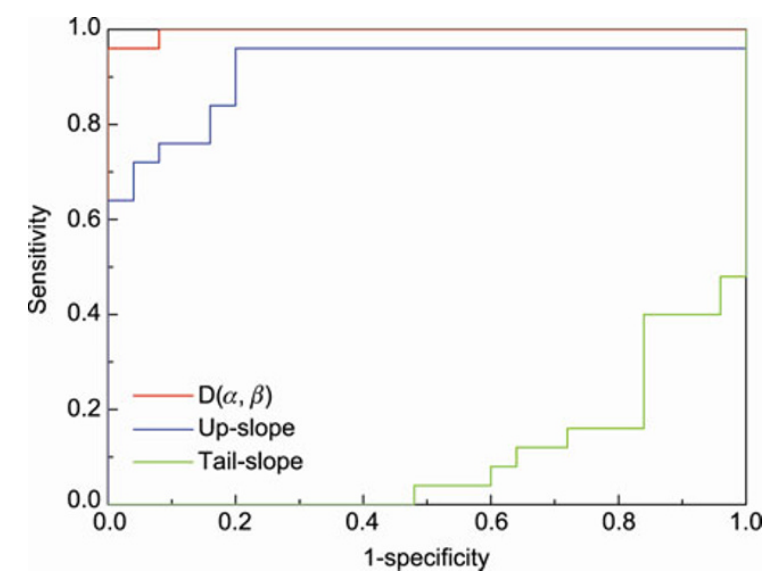

Figure 4 ROC curves obtained from the combined parameter of $\mathrm{D}(\alpha, \beta)$, up-slope and tail-slope in the classification of benign and malignant lesions.

Table 2 Statistical evaluation of using $\mathrm{D}(\alpha, \beta)$, up-slope and tail-slope in the classification of benign (25 ROIs) and malignant (25 ROIs) lesions ${ }^{\text {a) }}$

\begin{tabular}{ccc}
\hline Measurement & Area under curve $\left(A_{\mathrm{z}}\right)$ & $P$-value \\
\hline$A_{\mathrm{z}}(\mathrm{D}(\alpha, \beta))$ & $0.996 \pm 0.004$ & $<0.001$ \\
$A_{\mathrm{z}}$ (up-slope) & $0.916 \pm 0.045$ & $<0.001$ \\
$A_{\mathrm{z}}$ (tail-slope) & $0.104 \pm 0.043$ & $<0.001$ \\
\hline
\end{tabular}

a) $P$-values calculated with $Z$-tests were used to identify the significant differences between the estimated $A_{z}$ and an $A_{z}$ value of 0.5 . 
tail-slope.

\section{Discussion}

DCE-MRI data, acquired before and after the injection of contrast agent to evaluate the vascular characteristics of tissues [27-30], have been widely applied in the early detection of breast cancer. A dual S-shaped logistic model that is able to simulate the decaying phase was constructed in this retrospective study. It effectively quantified the breast lesion's kinetic curves based on DCE-MRI data and distinguished between benign and malignant breast lesions.

The enhancement mechanism of breast DCE-MRI is based on the paramagnetism of contrast agents. Such agents cause an initial increase in $\mathrm{T} 1$ and $\mathrm{T} 2$ relaxation rates of bulk water protons that is directly proportional to the concentration of contrast agents. After the initial increase, different patterns of contrast enhancement are demonstrated that reflect various histopathologic properties of the breast lesion, including vascular permeability factor (VPF), vascular endothelial growth factor (VEGF) and extracellular fluid volume [31-33]. In particular, VEGF, which is secreted by malignant lesions, is directly related with neoangiogenesis, bringing more blood supply in malignant lesions than benign lesions. Higher water content in tumor tissues, as well as their higher capillary permeability induced by underdeveloped blood vessel endothelium, contributes to a faster water exchange and diffusion rate [34-36]. As a result, concentration of contrast agent in malignant tissues decreases (washout phase) after an initially rapid growth during the measured time course, whereas the signal in benign lesions presents a persistent increase or plateau.

For the aforementioned reasons, DCE-MRI kinetic data are closely related with pathologic characteristics of breast lesions. Previous studies [3-12] have demonstrated that effective quantification of DCE-MRI data is crucial to diagnosis, inspection, and treatment assessment of breast cancer in clinic. In that sense, a subjective evaluation tool that retrieves features from DCE-MRI kinetic data for lesion classification is strongly favored.

Among the available feature extraction configurations based on the shape of lesion's kinetic curves, pharmacokinetics models were first demonstrated [13-15], which evaluate physiological parameters of lesion tissue from contrast agent concentration, converted from the signal intensity of images. Generally, pharmacokinetics models are composed of three parts: the AIF, the arterial output function (contrast agent concentration vs. time), and the simulation function. Embedded inaccuracies of this model result from uncertainties associated with AIF estimation and T1 baseline value. Many attempts have been made to reduce errors by imposing different assumptions, e.g., regulating localized AIF [34], but variances among different models cannot be ignored.

In contrast to the pharmacokinetic models, kinetic curve fitting based feature extraction does not require any input variables such as T1 baselines. Instead, the features for differentiation between benign and malignant lesions are extracted from the fitted curve. A recent study found [22] that the change of signal intensity is directly related with the metabolism of contrast agents in tissue, which can be simulated by a modified logistic regression model. In fact, a classic logistic model was originally a probability prediction method describing the system with binary response (e.g., death or survival) affected by multiple explanatory variables. It is capable of simulating a system's evolution with a single S-shaped (sigmoid) output, such as tumor and microbial growth [35-37], but fails to predict the washout phase in kinetic curves in DCE-MRI. The modified logistic regression model (eq. (5)) proposed by Moate et al. [22] includes an additional structure $\left(p_{5} \cdot t\right)$ in the standard logistic function (eq. (6)) to fit signal intensity curves with a decreasing terminal slope, which is able to predict a diverse range of gadolinium enhancement patterns.

$$
\begin{aligned}
& S I(t)=\frac{p_{2}+\left(p_{5} \cdot t\right)}{\left\{1+\exp \left(-p_{4} \cdot\left(t-p_{3}\right)\right)\right\}}+p_{1}, \\
& S I(t)=\frac{p_{2}}{\left\{1+\exp \left(-p_{4} \cdot\left(t-p_{3}\right)\right)\right\}}+p_{1} .
\end{aligned}
$$

Although no quantitative results were given to separate malignant lesions from benign lesions, $p_{2}, p_{4}$ and $p_{5}$ were extracted to demonstrate the possibility of using logistic equations for DCE-MRI data analysis.

In this work, as a step forward, a dual S-shaped logistic model with two diagnostic features, $\alpha$ and $\beta$, was developed ( $m$ and $n$ are scaling factors without diagnostic values). It not only effectively predicted the wash-out phase of the enhancement curves, but also well distinguished between malignant and benign lesions by using the derived diagnostic parameter $\mathrm{D}(\alpha, \beta)$.

In this model, the signal pattern that occurs immediately following the rapid initial increase is a direct consequence of the competition between $\alpha$ and $\beta$ as two opposing aspects of the impact on a kinetic curve's shape (shown in Figure 5).

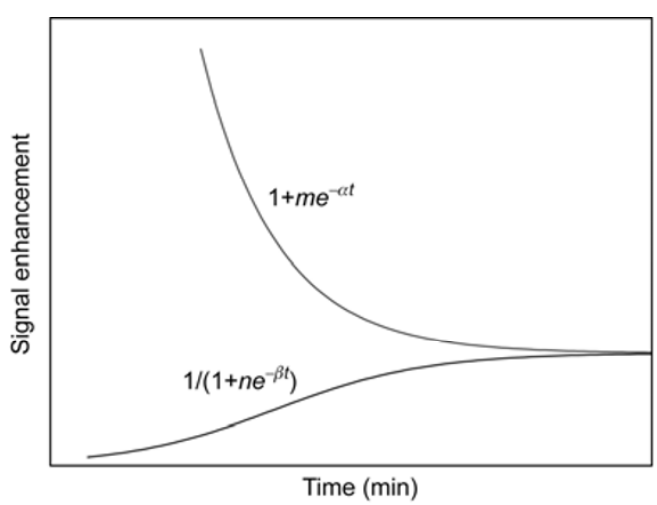

Figure 5 Schematic drawing of the influence of the two opposite aspects of the dual S-shaped logistic model on the shape of kinetic curves. 
In the enhancement pattern of typical benign tissues, the signal intensity continuously increases (type I). The parameter $\beta$ reflects the continuous growth of the signal. For typical malignant tissues, the signal intensity drops down after an initial increase (type III). The decrease of signal intensity can be described by $\alpha$. In other situations, a plateau phase is shown as a result of the balanced forces on both sides (type II).

However, since $\alpha$ and $\beta$ represent two opposing aspects of the characteristics of a breast lesion, for a more comprehensive description, both $\alpha$ and $\beta$ were employed as features to differentiate between benign and malignant breast lesions. Most notably, FLD could separate malignant lesions from benign lesions in parameter space, showing the potential diagnostic value of 2-D features ( $\alpha$ and $\beta$ ). In addition, the extraction procedure of $\alpha$ and $\beta$ is simple and straightforward.

Although favorable classification results were obtained in this study, limitations persist regarding ROI selection. Three ROI analysis methods are generally applied in DCE-MRI data analysis [38]: The first method employs a user-defined trace around the contour of the whole lesion and is associated with significant observer variability and bias; the second employs pixels with intensity values within $10 \%$ of the maximum; the third one, which was implemented in this study, selects a 9-pixel square in the most enhanced part of this region. Although small-sized ROIs improve image analysis efficiency, they also introduce lower SNR when characterizing the kinetics of the lesion. Furthermore, one small ROI may not be a reliable representation of the entire region, especially for heterogeneously enhanced lesions. In fact, there is not yet a consensus about the selection criterion [39]. Finally, it is noted that in this work the positioning of the ROI depends on a radiologists' visual assessment in choosing the most enhanced area, which may introduce subjective errors into clinical practice.

It is also worth mentioning that the number of benign and malignant lesions included in our study is limited, and should be improved in the future. In addition, Kuhl et al. [18] have found that there is some overlap between benign and malignant lesions in the three-type criteria caused by vascular distribution or subtypes with more complicated pathological characteristics. For instance, major subtypes of malignant lesions include invasive ductal carcinoma (IDC), ductal carcinoma in situ (DCIS) and invasive lobular carcinoma (ILC). For benign lesions, there are fibrocystic change (FCC), fibroadenoma, and papilloma [20]. In our research, the kinetic characteristics of these subtypes were not considered.

In conclusion, this retrospective study applied a dual S-shaped logistic model to fit the kinetic curves of breast lesions in DCE-MRI. Classification between benign and malignant lesions is achieved with sufficient accuracy by using the derived diagnostic parameter $\mathrm{D}(\alpha, \beta)$ superior to that of empirical parameters. The proposed method there- fore has potential for computer aided diagnosis in breast tumors. Preliminary results suggest that it also could be useful as a tool for breast cancer screening in the clinic.

We thank Zhong Hua for her assistance with modifications to the manuscript.

1 American Cancer Society. Cancer Facts \& Figures, 2009. Atlanta: American Cancer Society, Inc., 2009. 9-11

2 American Cancer Society. Breast Cancer Facts \& Figures 2009-2010. Atlanta: American Cancer Society, Inc., 2009. 2-8

3 Kneeshaw P J, Lowry M, Manton D, et al. Differentiation of benign from malignant breast disease associated with screening detected microcalcifications using dynamic contrast-enhanced magnetic resonance imaging. Breast, 2006, 15: 29-38

4 Bazzocchi M, Zuiani C, Painizza P, et al. Contrast-enhanced breast MRI in patients with suspicious microcalcifications on mammography: results of a multi-centre trial. AJR Am J Roentgenol, 2006, 186: 1723-1732

5 Hata T, Takahashi H, Watanabe K, et al. Magnetic resonance imaging for preoperative evaluation of breast cancer: a comparative study with mammography and ultrasound. J Am Coll Surg, 2004, 198: 190-197

6 Pickles M D, Lowry M, Manton D J, et al. Role of dynamic contrast-enhanced MRI in monitoring early response of locally advanced breast cancer to neoadjuvant chemotherapy. Breast Cancer Res Treat, 2005, 91: 1-10

7 Wasser K, Klein S K, Fink C, et al. Evaluation of neoadjuvant chemotherapeutic response of breast cancer using dynamic MRI with high temporal resolution. Eur Radiol, 2003, 13: 80-87

8 Padhani A R, Hayes C, Assersohn L, et al. Prediction of clinicopathological response of breast cancer to primary chemotherapy at contrast-enhanced MR imaging: initial clinical results. Radiology, 2006, 239: 361-374

9 Fischer U, von Heyden D, Vosshenrich R, et al. Signal characteristics of benign and malignant breast lesions in dynamic 2D-MRT of the breast. Fortschritte auf dem Gebiete der Rontgenstrahlen und der Neuen Bildgebenden Verfahren, 1993, 158: 287-292

10 Gibbs P, Liney G P, Lowry M, et al. Differentiation of benign and malignant sub-1 cm breast lesions using dynamic contrast-enhanced MRI. Breast, 2004, 13: 115-121

11 Goto M, Ito H, Akazawa K, et al. Diagnosis of breast tumors by contrast-enhanced MR imaging: comparison between the diagnostic performance of dynamic enhancement patterns and morphological features. J Magn Reson Imaging, 2007, 25: 104-112

12 Kelcz F, Santyr G E, Cron G O, et al. Application of a quantitative model to differentiate benign from malignant breast lesions detected by dynamic contrast-enhanced MRI. J Magn Reson Imaging, 1996, 6: $743-752$

13 Tofts P S, Kermode A G. Measurement of the blood-brain barrier permeability and leakage space using dynamic MR imaging. Magn Reson Med, 1991, 17: 357-367

14 Tofts P S, Brix G, Buckley D L, et al. Estimating kinetic parameters from dynamic contrast-enhanced T1-weighted MRI of a diffusable tracer: standardized quantities and symbols. J Magn Reson Imaging, 1999, 10: 223-232

15 Hassid Y, Furman-Haran E, Margalit R, et al. Noninvasive magnetic resonance imaging of transport and interstitial fluid pressure in ectopic human lung tumors. Cancer Res, 2006, 66: 4159-4166

16 Reed R K, Rubin K. Transcapillary exchange: role and importance of the interstitial fluid pressure and the extracellular matrix. Cardiovasc Res, 2010, 87: 211-217

17 Abdullah N, Mesurolle B, El-Khoury M, et al. Breast imaging reporting and data system lexicon for US: interobserver agreement for assessment of breast masses. Radiology, 2009, 252: 665-672

18 Kuhl C K, Mielcareck P, Klaschik S, et al. Dynamic breast MR im- 
aging: are signal intensity time course data useful for differential diagnosis of enhancing lesions? Radiology, 1999, 211: 101-110

19 Szabo B K, Aspelin P, Wiberg M K, et al. Dynamic MR imaging of the breast. Analysis of kinetic and morphologic diagnostic criteria. Acta Radiol, 2003, 44: 379-386

20 Jansen S A, Fan X, Karczmar G S, et al. Differentiation between benign and malignant breast lesions detected by bilateral dynamic contrast-enhanced MRI: a sensitivity and specificity study. Magn Reson Med, 2008, 59: 747-754

21 Heiberg E V, Perman W H, Herrmann V M, et al. Dynamic sequential 3D gadolinium-enhanced MRI of the whole breast. Magn Reson Imaging, 1996, 14: 337-348

22 Moate P J, Dougherty L, Schnall M D, et al. A modified logistic model to describe gadolinium kinetics in breast tumors. Magn Reson Imaging, 2004, 22: 467-473

23 Lagarias J C, Reeds J A, Wright M H, et al. Convergence properties of the nelder-mead simplex method in low dimensions. SIAM J Optimiz, 1998, 9: 112-147

24 Knopp M V, Weiss E, Sinn H P, et al. Pathophysiologic basis of contrast enhancement in breast tumors. J Magn Reson Imaging, 1999, 10: 260-266

25 Kinkel K, Hylton N M. Challenges to interpretation of breast MRI. J Magn Reson Imaging, 2001, 13: 821-829

26 Bone B, Wiberg M K, Parrado C, et al. Mechanism of contrast enhancement in breast lesions at MR imaging. Acta Radiologica, 1998, 39: 494-500

27 Buckley D L, Drew P J, Mussurakis S, et al. Microvessel density in invasive breast cancer assessed by dynamic Gd-DTPA enhanced MRI. J Magn Reson Imaging, 1997, 7: 461-464

28 Padhani A R. Dynamic contrast-enhanced MRI studies in human tumours. Br J Radiol, 1999, 72: 427-431

29 Parker G J, Suckling J, Tanner S F, et al. Probing tumor microvascu- larity by measurement, analysis and display of contrast agent uptake kinetics. J Magn Reson Imaging, 1997, 7: 564-574

30 Roberts H C, Roberts T P, Brasch R C, et al. Quantitative measurement of microvascular permeability in human brain tumors achieved using dynamic contrast-enhanced MR imaging: correlation with histologic grade. AJNR Am J Neuroradiol, 2000, 21: 891-899

31 Donahue K M, Weisskoff R M, Burstein D. Water diffusion and exchange as they influence contrast enhancement. J Magn Reson Imaging, 1997, 7: 102-110

32 Larsson H B, Rosenbaum S, Fritz-Hansen T. Quantification of the effect of water exchange in dynamic contrast MRI perfusion measurements in the brain and heart. Magn Reson Med, 2001, 46: 272-281

33 Buckley D L. Letter to the editor: transcytolemal water exchange and its effect on the determination of contrast agent concentration in vivo. Magn Reson Med, 2002, 47: 420-421

34 Calamante F, Morup M, Hansen L K. Defining a local arterial input function for perfusion MRI using independent component analysis. Magn Reson Med, 2004, 52: 789-797

35 Wang $\mathrm{H} \mathrm{N}$, Cui Y M, He H. A logistic model for magnetic energy storage in solar active regions. Res Astron Astrophys, 2009, 9: 687-693

36 Peleg M, Corradini M G, Normand M D. The logistic (Verhulst) model for sigmoid microbial growth curves revisited. Food Res Int, 2007, 40: 808-818

37 Sakuma Y, Okamoto N. A logistic regression predictive model and the outcome of patients with resected lung adenocarcinoma of $2 \mathrm{~cm}$ or less in size. Lung Cancer, 2009, 65: 85-90

38 Mussarakis S, Buckley D L, Horsman A. Dynamic MRI of invasive breast cancer: assessment of three region-of-interest analysis methods. J Comput Assist Tomogr, 1997, 21: 431-438

39 Gribbestad I S, Nilsen G, Fjosne H E, et al. Comparative signal intensity measurements in dynamic gadolinium-enhanced MR mammography. J Magn Reson Imaging, 1994, 4: 477-480

Open Access This article is distributed under the terms of the Creative Commons Attribution License which permits any use, distribution, and reproduction in any medium, provided the original author(s) and source are credited. 